\title{
Editorial
}

Miquel Taron, Adrián Llerena*, Vangelis G. Manolopoulos, Cristina Rodriguez-Antona, Sanja Stankovic and Ron H. N. van Schaik

\section{The need of the clinical implementation of pharmacogenetics in European health services for routine drug prescription. What's next? An urgent clinical unmet need for patients}

https://doi.org/10.1515/dmdi-2020-0172

Published online November 12, 2020

Keywords: drug prescription; health services; implementation; pharmacogenetics; pharmacogenomics; regulation.

More than 300 scientists and professionals from 42 countries, specialised in pharmacogenetics and precision medicine, met in Seville from the 16th to the 18th of October 2019, in the 5th International Congress of the European Society for Pharmacogenomics and Personalised Therapy (ESPT). In this meeting, organised in collaboration with the Spanish Society of Pharmacogenetics and Pharmacogenomics (SEFF), current aspects and future directions on research and clinical implementation of pharmacogenomics and personalised medicine were presented by world experts in this topic. The event brought together researchers and clinicians to discuss basic, clinical and translational research.

The theme of the ESPT 2019 congress was "Precision Medicine and Personalised Health" and the topics covered the latest developments in the field of pharmacogenetics/pharmacogenomics analysis on germline variants to liquid biopsy cell-free DNA in cancer patients to guide drug treatment. The congress keynote speakers included Prof. Dr. Sir Munir Pirmohamed, Prof. Dr. Ingolf Cascorbi, Dr. Lili Milani, Prof. Dr. Wolfgang Sadee, Prof. Dr. Daniel Mueller, Prof. Dr. Mike Caulfield and Prof. Dr. Klaus Pantel, among many other excellent speakers, and a prize was awarded by the evaluation committee to the best poster contribution among the 100 posters presented.

\section{Pharmacogenetics implementation in Spain and Europe}

\section{A joint declaration of ESPT and SEFF}

During the conference, the executive boards of ESPT and SEFF met and evaluated the degree of implementation of Pharmacogenetics and Personalised Medicine in the European Health Services. Both societies, supported and in representation of their respective members, agreed upon the following six specific points:

(1) Scientific evidence: There is a growing scientific evidence supporting the use of pharmacogenetics to improve the safety and efficacy of drug treatments.

(2) Drug regulation: The number of genetic biomarkers included by the Drug Regulatory Agencies in the Summary of Product Characteristics is constantly increasing (EMA 34\%, Spanish AEMPS >50\%) and has important clinical consequences.

(3) Research framework: European and National Research funding calls and programmes include Pharmacogenomics and Personalised Medicine as a priority.

(4) Policy makers: Awareness on the need of implementing pharmacogenetics and personalised medicine actions is increasing among policy makers. There are several initiatives in Europe; in Spain, the Senate has delivered a proposal asking for a National Strategy for Personalised Genomic Medicine.

(5) Current initiatives: There are initiatives that use pharmacogenetic markers, not only in individual clinical services and hospitals, but also at global public health services level (e.g. in Spain in Galicia, Catalonia, 
Andalusia and Extremadura) aimed at evaluating pharmacogenetics as Health Technology Assessment.

(6) Initiatives ESPT/SEFF: For the harmonisation of pharmacogenetics at different levels (laboratory proficiency testing, clinical guidelines, regulation, etc.), we ask for an interdisciplinary coordination effort.

Based on these points, ESPT and SEFF agreed on recommending that Pharmacogenetics and Personalised Medicine must be implemented at the routine clinical care at the European Health Services, in order to decrease patient and family suffering, but also health care costs, and contribute to sustainability of public health services across Europe.

Acknowledgments: MT, ALL, CR-A and RvS, VGM, SS were President, President-elect and Secretary of SEFF and ESPT respectively. The contribution of remaining board members of SEFF (Luis López, Francisco Abad, María Jesús Arranz, Anna González-Neira, Salvador Aliño) and ESPT (Maurizio Simmaco, Marc Ansari, Ingolf Cascorbi, Peter Jacobs, Janja Marc, Csilla Sipeky and Sofia Siest) is highly appreciated. Research funding: None declared.

Author contributions: All authors have accepted responsibility for the entire content of this manuscript and approved its submission.

Competing interests: Authors state no conflict of interest.
*Corresponding author: Adrián Llerena, MD PhD, SEFF Spanish Society of Pharmacogenetics and Pharmacogenomics (SEFF), Valladolid, Spain; ESPT European Society for Pharmacogenomics and Personalised Therapy (ESPT); and INUBE Extremadura Biosanitary University Research Institute, Pharmacogenetics and Personalized Medicine Unit, Badajoz University Hospital, Spain, E-mail: allerena@unex.es. https://orcid.org/0000-0002-5663-7081 Miquel Taron, SEFF Spanish Society of Pharmacogenetics and Pharmacogenomics (SEFF), Madrid, Spain; and Synlab Diagnósticos Globales SAU, Alcobendas (Madrid), Spain

Vangelis G. Manolopoulos, ESPT European Society for Pharmacogenomics and Personalised Therapy (ESPT), Milano, Italy; and Laboratory of Pharmacology, Medical School, Democritus University of Thrace, and Clinical Pharmacology and Pharmacogenetics Unit, Academic General Hospital of Evros, Alexandroupolis, Greece

Cristina Rodriguez-Antona, SEFF Spanish Society of Pharmacogenetics and Pharmacogenomics (SEFF), Madrid, Spain; Hereditary Endocrine Cancer Group, Spanish National Cancer Research Centre (CNIO), Madrid, Spain; and ISCIII Center for Biomedical Research on Rare Diseases (CIBERER), Madrid, Spain

Sanja Stankovic, ESPT European Society for Pharmacogenomics and Personalised Therapy (ESPT), Milano, Italy; and Center for Medical Biochemistry, Clinical Center of Serbia, Belgrade, Serbia

Ron H. N. van Schaik, ESPT European Society for Pharmacogenomics and Personalised Therapy (ESPT), Milano, Italy; and Department Clinical Chemistry, Erasmus MC University Medical Center, Rotterdam, The Netherlands 